\title{
Optimization of mass spectrometry settings for steroidomic analysis in young and old killifish
}

\author{
Rahel Dabrowski $^{1} \cdot$ Roberto Ripa $^{1} \cdot$ Christian Latza $^{1} \cdot$ Andrea Annibal $^{1} \cdot$ Adam Antebi $^{1,2}$
}

Received: 27 January 2020 / Revised: 2 March 2020 / Accepted: 3 April 2020 / Published online: 24 April 2020

(C) The Author(s) 2020

\begin{abstract}
Steroids are essential structural components of cell membranes that organize lipid rafts and modulate membrane fluidity. They can also act as signalling molecules that work through nuclear and $G$ protein-coupled receptors to impact health and disease. Notably, changes in steroid levels have been implicated in metabolic, cardiovascular and neurodegenerative diseases, but how alterations in the steroid pool affect ageing is less well understood. One of the major challenges in steroidomic analysis is the ability to simultaneously detect and distinguish various steroids due to low in vivo concentrations and naturally occurring stereoisomers. Here, we established such a method to study the mass spectrometry behaviour of nine sterols/steroids and related molecules (cholesterol precursors: squalene, lanosterol; sterol metabolites; 7 Dehydrocholesterol, 24, 25 and 27 Hydroxycholesterol; and steroids: progesterone, testosterone, and corticosterone) during ageing in the African turquoise killifish, a new model for studying vertebrate longevity. We find that levels of all tested steroids change significantly with age in multiple tissues, suggesting that specific steroids could be used as biomarkers of ageing. These findings pave the way for use of Nothobranchius furzeri as a novel model organism to unravel the role of sterols/steroids in ageing and age-related diseases.
\end{abstract}

Keywords Mass spectrometry $\cdot$ Orbitrap $\cdot$ Killifish $\cdot$ Steroidomics $\cdot$ Ageing

\section{Introduction}

Sterols and steroids are essential heterocyclic molecules used by all metazoans for diverse biological processes. They are important structural components of cell membranes that comprise lipid rafts and decrease membrane fluidity [1]. They also act as signalling molecules that typically work through nuclear and $\mathrm{G}$ protein-coupled receptors to regulate metabolism,

Electronic supplementary material The online version of this article (https://doi.org/10.1007/s00216-020-02640-6) contains supplementary material, which is available to authorized users.

Andrea Annibal

aannibal@age.mpg.de

$\bowtie$ Adam Antebi

aantebi@age.mpg.de

1 Max Planck Institute for Biology of Ageing, Joseph-Stelzmann-Strasse 9 b, 50931 Cologne, Germany

2 Cologne Excellence Cluster on Cellular Stress Responses in Aging-Associated Diseases (CECAD), University of Cologne, Josef-Stelzmann-Strasse 26, 50931 Cologne, Germany development, and homeostasis, [2] and their dysregulation can lead to disease.

The most abundant sterol is cholesterol, which is salvaged from the diet or de novo synthesized through a series of steps. A critical step in cholesterol synthesis is the cyclization of squalene to lanosterol, which can then be converted into cholesterol through multiple parallel pathways, one of which is via 7 Dehydrocholesterol [3]. Subsequently, cholesterol can undergo several modifications and transmutation reactions leading to a plethora of sterol/steroid-like molecules with different biological functions including oxysterols, bile acids, steroids, corticoids, and secosteroids [4].

Dysregulation of cholesterol synthesis and steroid levels in different tissues are associated with oxidative stress and cell death, which can contribute to age-related diseases and pathological conditions $[5,6]$. For example, increased levels of oxysterols have been implicated in Alzheimer's disease (AD), atherosclerosis, type 2 diabetes, and cancer [7-9]. In addition, in mice and humans blood steroid concentration changes with age, [10-12] and longitudinal studies have identified a correlation between age-related decline in steroid hormones and metabolic syndrome (MetS) as well as cardiovascular disease (CVD) $[13,14]$. 
Given the importance of various steroids in physiology and age-related disease it is essential to clarify their role in vivo, and dissect how their abundance and regulation changes with age. However, such efforts are currently hampered by the fact that vertebrate steroids are found at very low concentrations in vivo varying from 0.5 to $50 \mathrm{pg} / \mathrm{mL}$ in different tissues [15, 16], making it difficult to obtain enough material for extraction. Second, steroids often exist as stereoisomers and methods are lacking to separate and identify these distinct moieties. Third, analysing steroid levels in longitudinal studies remains challenging in humans. To overcome these problems, we established a model system using the African turquoise killifish and high-resolution mass accuracy mass spectrometry, which enables the detection of steroids in vivo and also allows us to reliably investigate their role in ageing. The African turquoise killifish Nothobranchius furzeri (N. furzeri) is an emerging model for the study of ageing and age-related diseases $[17,18]$. N. furzeri lives only 6 to 7 months, and is among the shortest-lived vertebrates that can be kept in laboratory conditions [19]. They display many features of human ageing, including loss of mobility, cancer, and cognitive decline [20, 21]. Notably, in killifish steroidogenesis occurs primarily in gonads, adrenal glands, liver, and brain [22-24]. Fish also typically contain high concentrations of steroids, facilitating the study of steroids in vivo [25-27].

Another important consideration is the ability to measure different biologically relevant steroid species in parallel. Traditionally, gas chromatography-mass spectrometry (GCMS) has been used for oxysterol analysis in cells, tissues, and plasma [28, 29]. GC-MS methods provide high information content, but require extensive sample preparation, making it a laborious option for routine or repeated analyses. As an alternative, electrospray ionization (ESI) coupled to liquid chromatography-mass spectrometry (LC-MS) is the preferred method for high-throughput studies and reverse-phase LCMS has been extensively employed for the analysis of estradiol, testosterone, and corticosterone in human urine, plasma and serum [30-32]. However, none of the currently described methods are able to detect more than six steroids of interest with high sensitivity $[15,33]$.

In the current study, we thus established a highly sensitive high-resolution mass spectrometry-based method to measure nine different steroids in the killifish $N$. furzeri during the ageing process.

\section{Material and methods}

Chemicals UHPLC-grade water, UHPLC-grade acetonitrile, and formic acid were purchased from Biosolve Valkenswaard, Netherlands. UHPLC methanol and ethyl acetate were purchased from Sigma-Aldrich, GmbH. Isopropanol was purchased from Carl Roth $\mathrm{GmbH}$ und Co. KG
(Karlsruhe, Germany) and Chloroform was purchased from Merck KGaA (Darmstadt, Germany). The following standards were obtained from Sigma-Aldrich, GmbH: 7 Dehydrocholesterol, 24(S) Hydroxycholesterol, 25 Hydroxycholesterol, 27 Hydroxycholesterol, corticosterone, lanosterol, progesterone, squalene, 7-Ketocholesterol (d7), and testosterone as well as tricaine methanesulfonate.

LC-MS instrumentation LC-MS analysis was carried out on a Q-Exactive Plus, (Thermo Fischer Scientic GmbH, Bremen, Germany). Steroid standards were separated using a XSelect

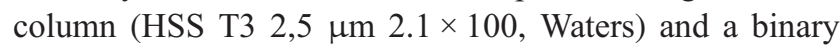
pump system (Vanquish, ThermoFisher Scientific GmbH, Bremen, Germany) with solvent $\mathrm{A}$ as water with $0.1 \% \mathrm{v} / \mathrm{v}$ formic acid and eluent $B$ as acetonitrile with $0.1 \% \mathrm{v} / \mathrm{v}$ formic acid. The gradient started with $10 \%$ eluent $\mathrm{B}$ and kept for 0.3 min., then ramped to $99 \%$ eluent B in 8 min and held for $2 \mathrm{~min}$. in $1 \mathrm{~min}$ it decreased to $10 \%$ eluent B and kept it for $1 \mathrm{~min}$. The total time for the gradient was $12 \mathrm{~min}$ and the column was kept at $45^{\circ} \mathrm{C}$.

To narrow down the crucial settings to maximize the sensitivity for the quantification of steroid in killifish samples, we explored several mass spectrometry parameters using steroid standards (100 ng). Spray voltage was set from 1 to $6 \mathrm{kV}$ in positive ion mode. Then, mass resolving power 17.500, 30.000, 70.000, 140.000, and 280.000 were compared and automated gain control (AGC) settings $2 \mathrm{e}^{4}, 5 \mathrm{e}^{4}, 1 \mathrm{e}^{5}, 2 \mathrm{e}^{5}, 5 \mathrm{e}^{5}, 1 \mathrm{e}^{6}, 3 \mathrm{e}^{6}$, and $5 \mathrm{e}^{6}$ were tested. Lastly, injection time (IT) 100, 150, 200, and $250 \mathrm{~ms}$ were examined, while sheet gas and auxiliary gas were kept constant at 20 and 5 respectively as well as S-lenses at 60 (a.u.).

We then examine the following liquid chromatography parameters for optimal steroid identification: flow rates 50, 100, 150 , and $200 \mu \mathrm{L} / \mathrm{min}$ as well as needle positions A, B, C, and $\mathrm{D}$ from the mass spectrometer inlet.

During the analysis we monitored the following ions: squalene->411.3985, lanosterol->427.3934, 7 Dehydrocholesterol- $>385.3465,24-25-$ and 27 Hydroxyholesterol- $>403.3571$, progesterone- $>315.2319$, corticosterone- $>347.2217$, testosterone- $>289.2162$.

Data was analysed using Xcalibur version 4.0 and Trace Finder version 4.1 .

Killifish husbandry and tissue collection All experiments were performed using the $N$. furzeri short-lived strain GRZ-AD. Following fertilization, eggs were collected by sieving the sand with a plastic net and kept in wet peat moss during embryonic development. Embryos were then hatched by flushing the peat with tap water at $16-18{ }^{\circ} \mathrm{C}$ and transferred to a clean vessel with a cut plastic pipette. Fry were fed with newly hatched Artemia nauplii for the first 2 weeks and then weaned 


\section{Cholesterol Biosynthesis}

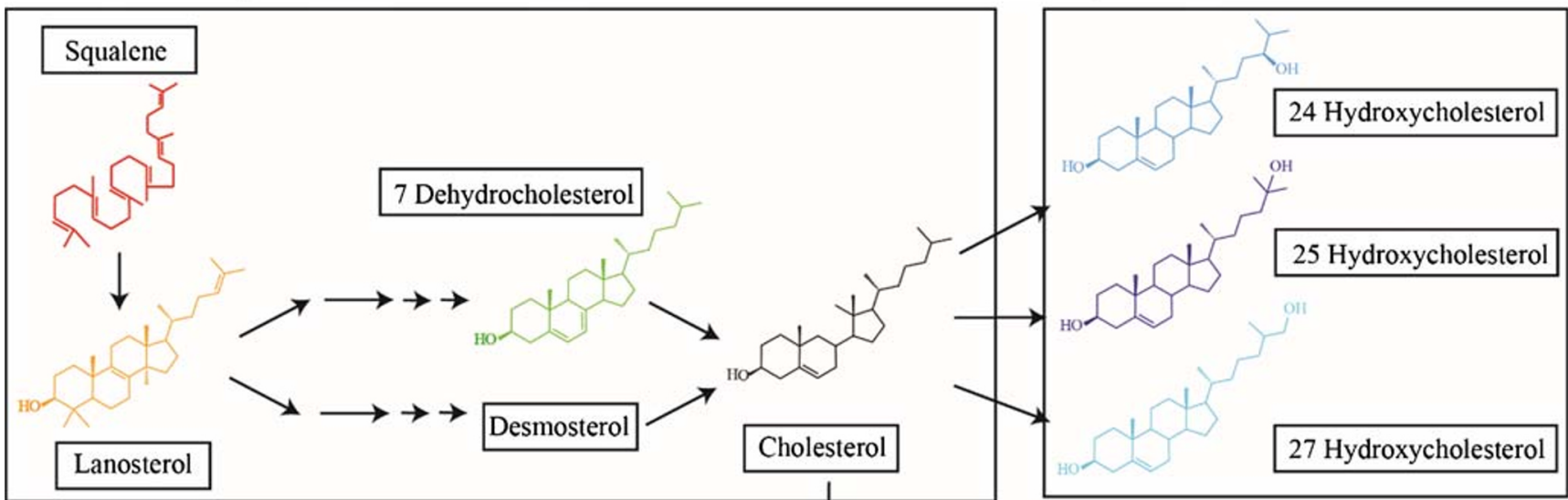

\section{Androgens}

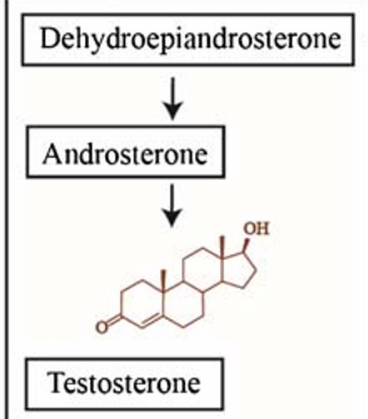

\section{Oxysterols}

\section{Mineralocorticoids}

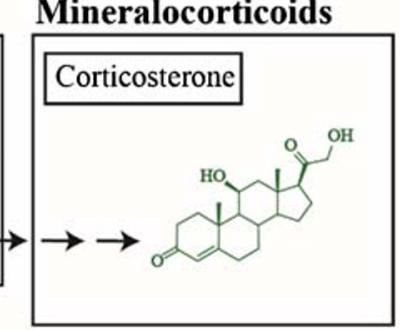

Scheme 1 Chemical structures of the main steroids. Steroids are shown in groups (Cholesterol Biosynthesis, Oxysterols, Progestagens, Mineralcorticoids and Androgens) and in order of synthesis. Colours of chemical structures are identical with colours of data points in the result figures

with finely chopped Chironomus larvae. Subsequently, fish were raised individually in 2.8 - L tanks at $27{ }^{\circ} \mathrm{C}$ and fed two times a day with frozen Chironomus larvae or living nauplii of Artemia salina. For tissue collection, fish were sacrificed using an overdose of tricaine methanesulfonate (TMS, MS-222) $(0.4 \mathrm{mg} / \mathrm{mL})$ and brain, liver, gonads and gut tissue was immediately extracted by dissection. Tissues were frozen in liquid nitrogen and stored at $-80 \mathrm{C}^{\circ}$. For ageing analysis, we collected samples from six 5-week-old and six 15-weekold wild-type male fish.

Steroids extractions from killifish tissues Tissues underwent up to three thaw and freeze cycle in liquid nitrogen and were then homogenized by bead beatings for $30 \mathrm{~min}$ at 50 oscillations/s at $4{ }^{\circ} \mathrm{C}$ using the Qiagen tissue lyser. A volume corresponding to $100 \mu \mathrm{g}$ proteins was subjected to three different extraction methods, namely Bligh and Dye, methanol extraction, and solid-phase extraction (SPE). Before extraction, $2 \mathrm{ng}$ of internal standard (7-Ketocholesterol-d7) was added. In the first method, $150 \mu \mathrm{L}$ chloroform/methanol 2:1 $\mathrm{v} / \mathrm{v}$ was added to the samples, whereas in the second method, $100 \mu \mathrm{L}$ of cold methanol was added. In both methods, samples were afterwards rotated for $1 \mathrm{~h}$ at $4{ }^{\circ} \mathrm{C}$ and centrifuged for $10 \mathrm{~min}$ at $10.000 \mathrm{rpm}$. The supernatant was transferred to a new tube in both methods and dried using a speedvac as described in our previous works [34, 35]. The third method used SPE to enrich the sterol fraction: HLB prime cartridges (Oasis, Waters) were preconditioned with $1 \mathrm{~mL}$ methanol and $1 \mathrm{~mL}$ water with $1 \% \mathrm{v} / \mathrm{v}$ formic acid before adding the sample. After the sample was applied, phospholipids were removed by adding $1 \mathrm{~mL}$ hexane. Steroids were eluted using $500 \mu \mathrm{l}$ ethyl acetate as previously described [36]. The organic fraction was dried in a speed vac as described above. Twenty microliters of $50 \% \mathrm{v} / \mathrm{v}$ methanol/water was used to reconstitute the sample and $5 \mu \mathrm{L}$ was injected into the LC-MS/MS system. The relative response for each steroid species was calculated by dividing the peak area of the analyte to the internal standard peak area (7-Ketocholesterol-d7) and further normalized to protein concentration.

Statistics Statistical analyses were performed using GraphPad Prism software 7.03. $P$ values were calculated with $t$ test. 


\section{Results}

\section{Optimization of mass spectrometry settings}

We used ultra-high pressure liquid chromatography (UHPLC) coupled with electrospray mass spectrometry (ESI-MS) for the detection and identification of nine steroids (Scheme 1). Since steroids are present at low concentrations in vivo we first optimized different parameters to increase sensitivity. In all optimizations, we report the mean intensity for each standard in the figures, whereas the corresponding relative standard deviations (RSD) are reported in Electronic Supplementary Material (ESM) Tables (S1-S7). The measurements have a RSD ranging from $0.28 \%$ to $12-13 \%$.

The separation gradient was kept constant in all optimization experiments. Squalene eluted at $2.25 \mathrm{~min}$, lanosterol at $8.03 \mathrm{~min}, 7$ Dehydrocholesterol at $9.83 \mathrm{~min}$, progesterone at $7.48 \mathrm{~min}$, corticosterone at $5.93 \mathrm{~min}$, testosterone at $6.53 \mathrm{~min}$ (Table 1). Although the three oxysterols have the same $\mathrm{m} / \mathrm{z}$ value, they eluted at different times: 24 Hydroxycholesterol at $9.84 \mathrm{~min}, 25$ Hydroxycholesterol at 8.91, and 27 Hydroxycholesterol at $10.2 \mathrm{~min}$.

We initially investigated the effect of the spray voltage on nine standards (Fig. 1a). All nine species were detected from 1 up to $6 \mathrm{kV}$.

Ion intensity was shown to be proportional to the magnitude of the voltage. An increase of voltage leads to a stronger electric field and by consequence, a stronger force pulls the ion spray into the mass spectrometer. Oxysterols generally followed this expected pattern and while peak intensities were generally low, they increased with higher voltages. Similarly, lanosterol showed higher peak intensity at higher voltage. The other steroids/sterols (squalene, corticosterone, testosterone, progesterone and 7 Dehydrocholesterol, 24 Hydroxycholesterol) did not show this pattern and peak intensity either plateaued or decreased at higher spray voltage. However, higher voltages may also cause insource fragmentation. When $5 \mathrm{kV}$ and $6 \mathrm{kV}$ were used, the intensity of the ions decreases in comparison with $4 \mathrm{kV}$. We observed highest peak intensities for most species at a spray voltage of $4 \mathrm{kV}$ and thus performed all further optimizations with this voltage.

We next explored all available resolutions ranging from 17.500 to 280.000 (Fig. 1b). In general, high resolution is required for complex samples and in particular for the separation of stereoisomers. Nevertheless, high resolution can also lead to a decrease in sensitivity because it requires longer Orbitrap scan times. Peak intensities of the oxysterols, corticosterone and lanosterol did not differ greatly between the tested resolutions. Resolutions 17.500 and 70.000 resulted in slightly higher peaks for corticosterone and lanosterol. Testosterone peak intensity was also higher at 70.000 resolution and 7 Dehydrocholesterol could only be detected with a resolution of 70.000 . We therefore continued the optimization using the resolution at 70.000 .

In terms of ion population, we selected optimal automatic gain control (AGC) target values at $2 \mathrm{e}^{4}, 5 \mathrm{e}^{4}, 1 \mathrm{e}^{5}$, $2 \mathrm{e}^{5}, 5 \mathrm{e}^{5}, 1 \mathrm{e}^{6}, 3 \mathrm{e}^{6}$, and $5 \mathrm{e}^{6}$ (Fig. 1c). AGC controls the number of ions that will enter the Orbitrap. A higher AGC will cause space charge effects and decrease sensitivity. In addition, the scan rate is slowed as the AGC increases, which further affects the sensitivity of the measurement. Most steroids showed highest or second highest peak intensities with an AGC of $1 \mathrm{e}^{5}$. However, oxysterols could only be detected with AGCs $2 \mathrm{e}^{5}$ or higher. 25 and 27 Hydroxycholesterol could even only be detected with an AGC of $3 \mathrm{e}^{6}$. Consequently, we continued the parameter optimization using an AGC of $3 \mathrm{e}^{6}$.

We analysed peak intensities of the steroid standards at varying injection times (IT) while the resolution is kept constant at 70.000 (Fig. 1d). The IT can dramatically affect the sensitivity of the analysis, since it determines how long the ions can accumulate in the C-trap. Peak intensities for most steroids were by far the highest with an injection time of $200 \mathrm{~ms}$, whereas higher injection time $(250 \mathrm{~ms})$ decreased the intensities of all standards. We thus proceeded with $200 \mathrm{~ms}$.

Table 1 ESI-MS/MS linear parameters for steroids

\begin{tabular}{|c|c|c|c|c|c|c|c|}
\hline Compound & $\mathrm{m} / \mathrm{z}$ & $t_{\mathrm{R}}(\min )$ & LOD (pg/mL) & LOQ (pg/mL) & Slope & Intercept & $R^{2}$ \\
\hline Squalene & 411.3985 & 2.25 & 0.51 & 1.71 & $3.01 \mathrm{E}+04$ & $1.89 \mathrm{E}+06$ & 0.94 \\
\hline Lanosterol & 427.3934 & 8.03 & 0.32 & 1.06 & $2.57 \mathrm{E}+06$ & $1.79 \mathrm{E}+04$ & 0.99 \\
\hline 7 Dehydrocholesterol & 385.3465 & 9.83 & 0.19 & 0.62 & $4.99 \mathrm{E}+05$ & $9.86 \mathrm{E}+05$ & 0.90 \\
\hline 24 Hydroxyholesterol & 403.3571 & 9.84 & 1.00 & 9.79 & $1.83 \mathrm{E}+05$ & $2.74 \mathrm{E}+06$ & 0.94 \\
\hline 25 Hydroxycholesterol & 403.3571 & 8.91 & 2.63 & 3.57 & $4.07 \mathrm{E}+05$ & $1.74 \mathrm{E}+06$ & 0.99 \\
\hline 27 Hydroxyholesterol & 403.3571 & 10.2 & 3.59 & 4.56 & $5.47 \mathrm{E}+05$ & $8.11 \mathrm{E}+05$ & 0.99 \\
\hline Progesterone & 315.2319 & 7.48 & 2.94 & 3.34 & $4.98 \mathrm{E}+05$ & $4.57 \mathrm{E}+06$ & 0.91 \\
\hline Corticosterone & 347.2217 & 5.93 & 1.07 & 8.76 & $4.94 \mathrm{E}+05$ & $4.57 \mathrm{E}+06$ & 0.99 \\
\hline Testosterone & 289.2162 & 6.53 & 1.37 & 11.96 & $3.78 \mathrm{E}+05$ & $1.77 \mathrm{E}+06$ & 0.91 \\
\hline
\end{tabular}


a

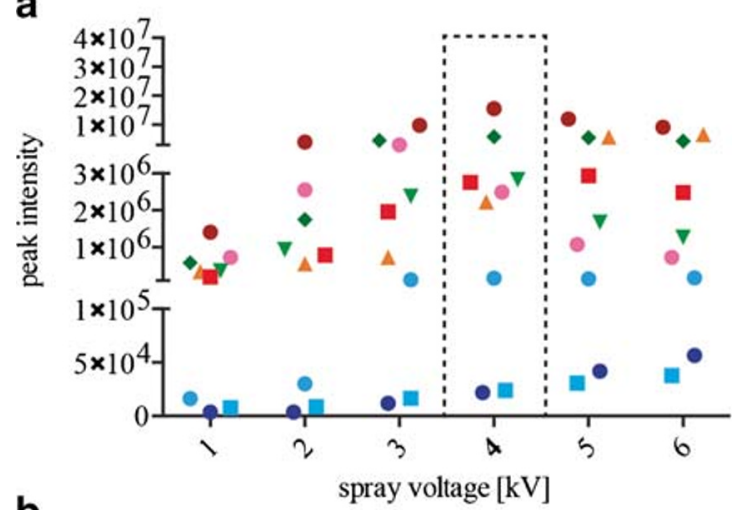

b

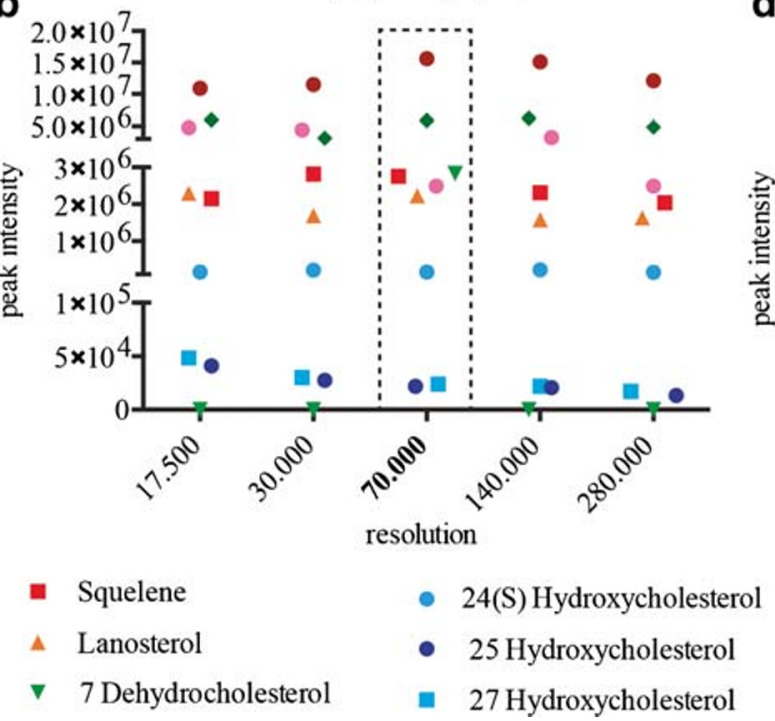

C
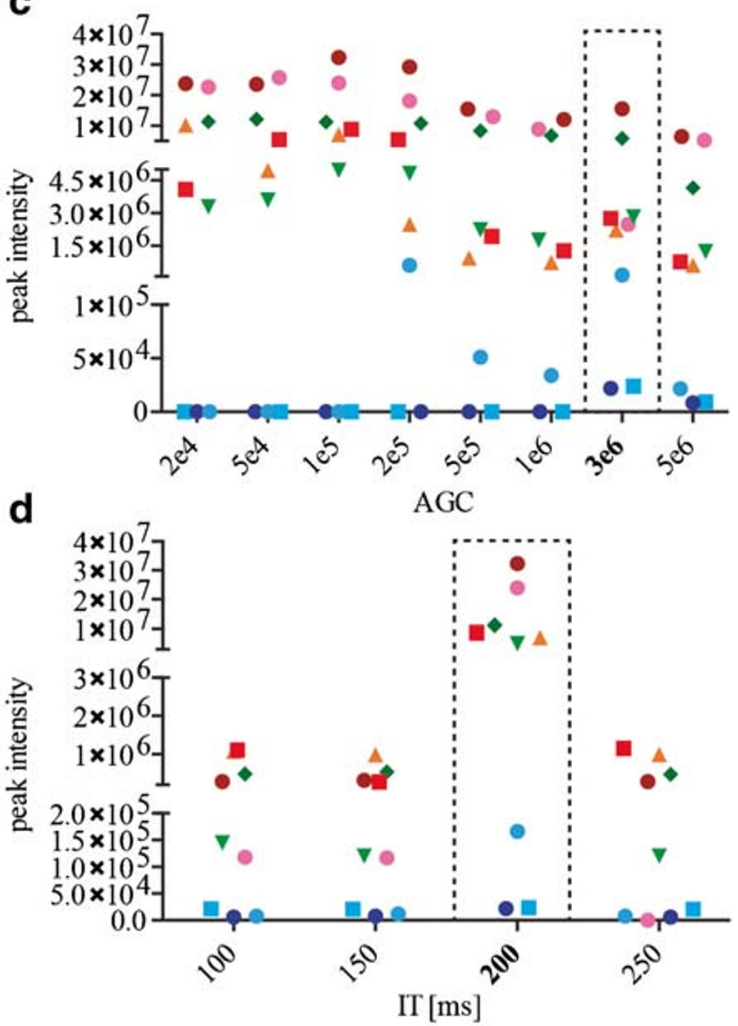

Fig. 1 Optimization of the MS parameters: MS parameters that were tested in this study. a Peak intensities of all tested compounds with different spray voltages. b Peak intensities of all tested compounds with all available resolutions. $\mathbf{c}$ Peak intensities of all tested compounds with

In our attempt to further optimize the MS settings, we tested two parameters of the liquid chromatography: flow rate and needle position (Fig. 2 a and b). The decrease of the mobile phase rate also decreases the droplet radius, which generally turns in an increase in sensitivity. However, we found that $50 \mu \mathrm{L} / \mathrm{min}$ (the slowest flow rate we tested) resulted in the lowest peak intensities. Peak intensities of oxysterols as well as squalene and 7 Dehydrocholesterol were highest with a flow rate of $150 \mu \mathrm{L} / \mathrm{min}$. Based on our results, we decided to carry on our optimization using a flow rate of $150 \mu \mathrm{L} / \mathrm{min}$.

Finally, we analysed changes in sensitivity by varying needle positions A, B, C, and D (Fig. 2b), which changes the distance between the electrospray needle and the MS inlet. With the exception of squalene, lanosterol and 24 Hydroxycholesterol position D, which results in the shortest distance between the needle and the inlet, provided the highest peak intensities and thus produced the best results for steroid identification overall. several AGC. d Injection times. Each data point is the mean value of an experimental triplicate. Relative standard deviations for all measurements are reported in ESM Tables S1-S4. Optimal conditions are indicated using a dotted box

\section{Method validation}

Using the optimizations described above we established an LC-MS method for steroid analysis with the following parameter settings: $4 \mathrm{kV}$ spray voltage, resolution of $70.000,3 \mathrm{e}^{6} \mathrm{AGC}, 200 \mathrm{~ms}$ IT, $150 \mu \mathrm{L} / \mathrm{min}$ flow rate and needle position $\mathrm{D}$. These settings were validated using serial dilutions of the nine standards from $0.001 \mathrm{nmol} / \mathrm{L}$ to $500 \mathrm{nmol} / \mathrm{L}$ (Tables 1 and 2). Intraday and interday variability were calculated for all steroid standards. RSD for both measurements were below $15 \%$ of variation. Standards were also spiked in killifish matrix prior or after SPE enrichment to calculate recovery rate. Detection limits (LOD) ranged from $0.19 \mathrm{pg} / \mathrm{mL}$ for 7 Dehydrocholesterol to $2.94 \mathrm{pg} / \mathrm{mL}$ for progesterone. Moreover, quantification limits (LOQ) ranged from 0.62 to $11.96 \mathrm{pg} / \mathrm{mL}$, which are two to three-fold improved compared to previous works (ESM Table S10) [30, 37, 38]. All external calibration curves exhibited high levels of linearity $(>0.90)$. The measurement using 


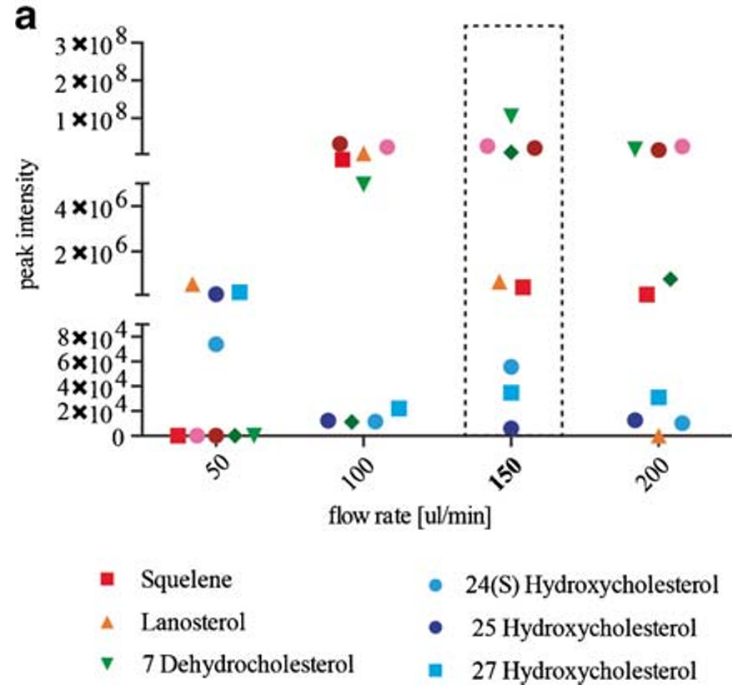

Fig. 2 Optimization of the ion source and liquid chromatography parameters: LC parameters that were tested in this study. a Peak intensities of all tested compounds with different flow rates. b Peak intensities of all tested compounds with needle positions A, B, C, or D.

Orbitrap allowed obtaining the elemental composition of each steroid using less than $5 \mathrm{ppm}$ mass accuracy (ESM Table S8).

\section{Steroid extraction and in vivo quantification of steroids in killifish}

After we established optimal LC-MS parameters for the detection of steroids, we next used the newly developed method to investigate the concentration of selected steroids in killifish tissues. For this we first optimized steroid extractions from tissue using wild-type killifish gonads. We analysed peak intensities of all nine steroids (Fig. 3). This showed that with the exception of lanosterol and corticosterone, the intensity for most species was highest using the SPE enrichment. SPE removes salts and other contaminants that can potentially influence the sensitivity of the measurement. Thus, we used

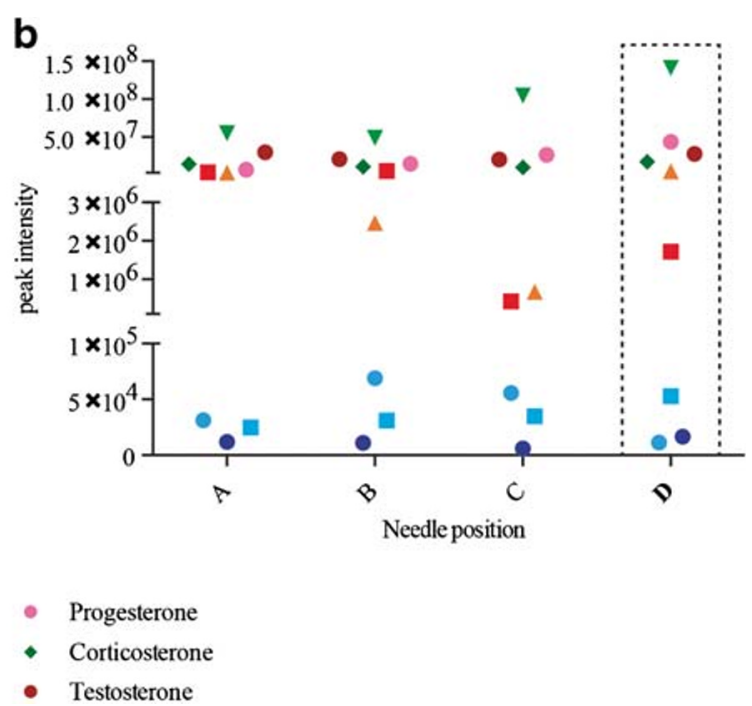

Each data point is the mean value of an experimental triplicate. Relative standard deviations for all measurements are reported in ESM Tables S5S6. Optimal conditions are indicated using a dotted box

SPE in combination with our new LC-MS method to extract and identify steroids from four different tissues, namely gut, liver, brain, and gonad in young (5-week-old ) and (15-weekold) killifish.

Our results are represented as heatmaps for all tissues (Fig. $4 \mathrm{a}-\mathrm{d}$ ) and histograms for individual steroids in each tissue are included in the ESM (Figs. S1-S4).

Overall, we found that the level of almost all steroids change with age, with the most change occurring in the gonad and the least change observed in the gut. 25 and 27 Hydroxycholesterol showed an increase in all tissues with age (with statistically significant increases in the liver and gonads), while all other steroids were unchanged or detected at lower quantities in aged tissues. Squalene and lanosterol concentrations were significantly reduced in old fish compared to young in all tested tissues apart from the gut, ranging between two-fold to four-fold of reduction (Fig. 4 b-d). 7
Table 2 Intraday, interday error and recovery of the LC-MS method

\begin{tabular}{lccc}
\hline Compound & Intraday precision $(n=5)$ & Interday precision $(n=5)$ & Recovery $(\%)$ \\
\hline & $\operatorname{ng} \pm \mathrm{SD}(\% \mathrm{RSD})$ & $\mathrm{ng} \pm \mathrm{SD}(\% \mathrm{RSD})$ & \\
7-Ketocholesterol $(\mathrm{d} 7)$ & $1.938 \pm 0.16(8.29)$ & $1.85 \pm 0.17(9.15)$ & 81.73 \\
Squalene & $0.544 \pm 0.07(12.97)$ & $1.106 \pm 0.083(7.52)$ & 91.93 \\
Lanosterol & $1.049 \pm 1.08(11.97)$ & $2.366 \pm 0.30(13.03)$ & 88.61 \\
7 Dehydrocholesterol & $0.780 \pm 0.05(6.80)$ & $2.011 \pm 0.28(14.01)$ & 94.18 \\
24 Hydroxyholesterol & $1.268 \pm 0.06(5.37)$ & $1.105 \pm 0.09(8.27)$ & 84.92 \\
25 Hydroxycholesterol & $1.077 \pm 0.05(5.12)$ & $1.75 \pm 0.20(11.68)$ & 86.25 \\
27 Hydroxyholesterol & $1.267 \pm 0.15(11.99)$ & $0.584 \pm 0.071(12.15)$ & 78.53 \\
Progesterone & $0.465 \pm 0.46(2.29)$ & $1.217 \pm 0.12(10.31)$ & 73.82 \\
Corticosterone & $1.097 \pm 1.16(10.7)$ & $0.816 \pm 0.083(10.26)$ & 93.81 \\
Testosterone & $0.915 \pm 0.12(13.59)$ & $1.961 \pm 0.15(7.83)$ & 81.00 \\
\hline
\end{tabular}




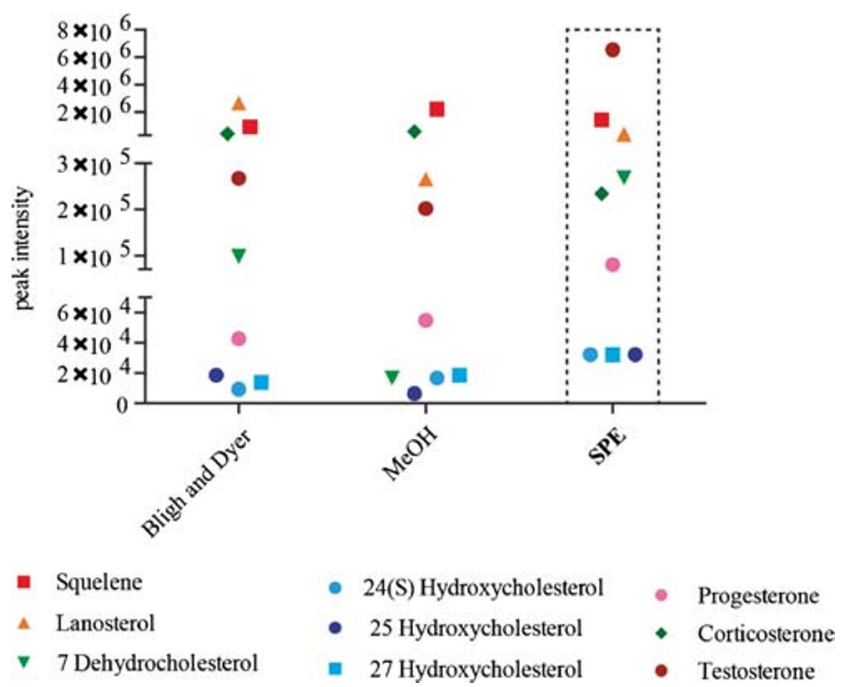

Fig. 3 Evaluation of three different methods for steroid extraction from killifish tissues. Peak intensities of all tested compounds using Bligh and Dyer, $\mathrm{MeOH}$ extraction or SPE. Each data point is the mean value of an experimental triplicate. Relative standard deviations for all measurements are reported in ESM Table S7. Optimal conditions are indicated using a dotted box

Dehydrocholesterol concentrations showed a tendency to be reduced in aged fish, but the reduction was only statistically significant in the liver (Fig. 4b). Quantification of 24 hydroxycholesterol levels showed an approximately twofold reduction in the brain of 15-week-old fish compared to 5 -week-old fish but their levels remained unchanged in gonads and the liver (Fig. 4b, d). Levels of progesterone and testosterone significantly decreased in gonads during ageing (Fig. 4d). In particular, progesterone levels were also reduced in other tissues (more than three-fold in the brain and about two-fold in the gut) in aged tissues (Fig. 4a, c), while testosterone and corticosterone levels showed less change in brain and gut tissues. However, they decreased significantly in the liver and gonads.

\section{Discussion}

In this study we developed a method for the detection of nine steroids from different tissues of the African turquoise killifish, $N$. furzeri, using LC-MS. The analysis of steroids remains a challenge due to their low concentrations in vivo and their structural similarities [39]. Our newly developed method, however, allows a sensitive detection and simultaneous quantification of nine steroids from different classes.

We optimized both technical parameters of the mass spectrometry and tissue extraction.

We observed a different mass spectrometric behaviour of the steroid standards while we were optimizing each parameter. When using higher voltages at the electrospray source needle, it is likely that discharge can occur between the needle and the counter electrode corona above certain potentials. This results in unstable spray and thereby decreases the signal intensity. However, this was observed only for few steroids, whereas the other steroids were less affected. Lubin A, and Leinonen A, already described this discrepancy, which is associated with the intrinsic physical-chemical properties of the compound and the presence of different function groups, such as 1,4-diene-3-one structure, hydroxyl groups, or carbonyl groups, which affects proton affinity and by consequences ionization efficiency of the different steroids $[40,41]$.

We also observed the differential behaviours of the steroids at different resolutions. The resolution is dependent on the scan time of the Orbitrap. The higher the resolution, the longer the time the Orbitrap needs to separate the ions, which may affect sensitivity (about $512 \mathrm{~ms}$ for resolution of 140,000). Nevertheless, this effect is not visible under our conditions. As described by Kalli A et al., a possible explanation could be, that the concentration of the standard mix is too high for the effect on the resolution to be seen [42], in particular at resolution 280.000. In this optimization, we used sub-molar concentrations of the standards, $100 \mathrm{ng}$, which is not very far from the physiological concentration (ranging from $0.5 \mathrm{pg} / \mathrm{mL}$ to $5 \mathrm{ng} /$ $\mathrm{mL}$ in brain and liver) and allowed an easy detection and identification of the steroid species (ESM Table S8).

The AGC target values also dramatically influence the sensitivity for the detection of the steroid standards. Knarchenko et al. investigated these effects using a simulation approach [43]. High AGC target values will result in a slower scan rate and possibly space charge effects, which negatively affect the sensitivity and mass accuracy. On the contrary, lower AGC values lead to less accumulation of ions in the C-trap, which results in lower sensitivity.

We show for the first time a different behaviour of steroid ions at different AGC values.

The reason why the oxysterols could be detected only from AGC values above $2 \mathrm{e}^{5}$ remains unclear. We can speculate that either the hydrophobicity of the compounds might have an effect during ion accumulation, or as described by Szabó PT et al., the hydrophobicity of the molecules can affect the ionization process and reduce the number of ions, which can further enter the mass spectrometer [44].

We also report for the first time the effect of injection time on the detection of steroid species using a constant resolution and AGC value. These three parameters are linked together and optimization needs to be done to assess which combination allows higher signal intensity for the desired molecules. As described previously by Michalski A et al., when the injection time is reached, the ions will be injected into the Orbitrap, regardless of whether the AGC target is reached [45]. We showed that the combination of AGC target $3 \mathrm{e} 6$ and $200 \mathrm{~ms}$ of injection time is beneficial for steroid detection and identification. Based on the concentration of steroid species we can speculate that most of the ions are accumulated in 


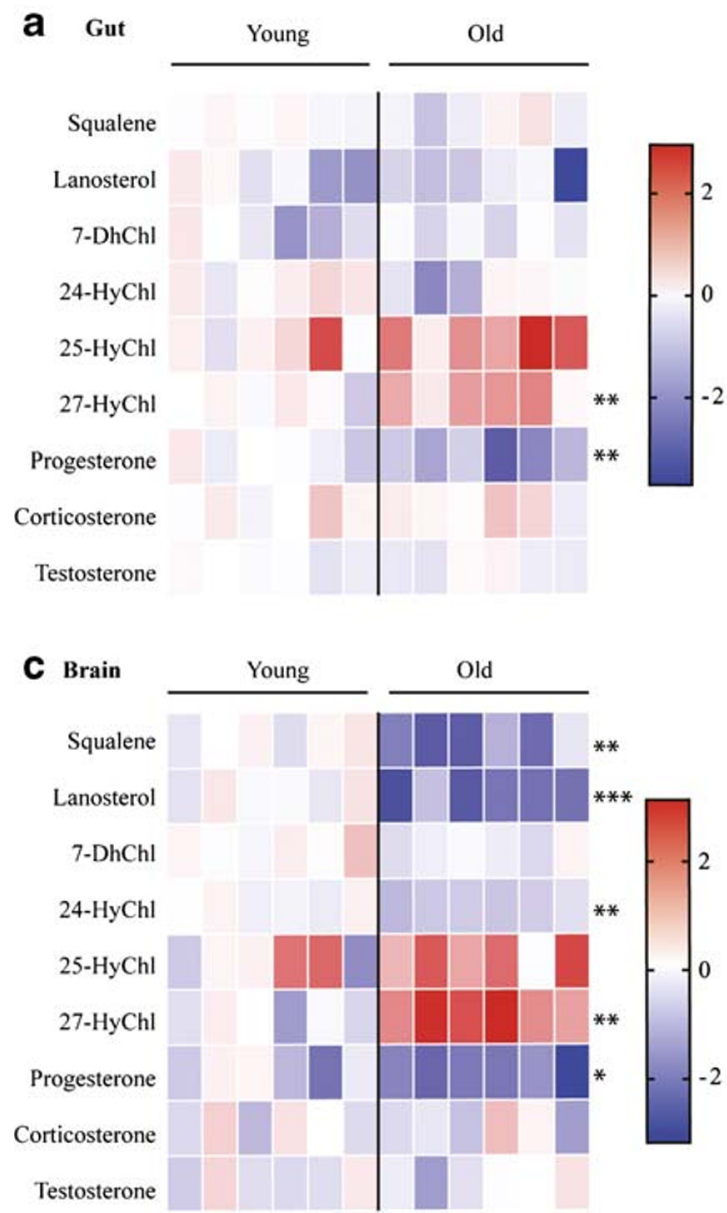

Fig. 4 In vivo concentrations of steroids in young and old killifish in the gut, liver, brain, and gonad. Heat map of steroid concentrations from gut (a), liver (b), brain (c), and gonads (d) of six young and six old male fish. Steroid concentrations are depicted on a $\log 10$ scale. High concentrations

the C-trap and reached the desired AGC values in a time frame that is very close to the selected injection time.

Steroids generally represent a small fraction of the total lipidome of cells and the organism and multiple analytical techniques to extract and enrich for steroids have been reported $[29,46]$. Our comparison of three extraction methodologies is in good agreement with previously published data. We show that solid-phase extraction (SPE) remains the golden standard for the purification and enrichment of most species. We additionally include a hexane wash to remove the hydrophobic lipids as suggested by Azadmard-Damirchi S. et al., which resulted in higher intensity and increased sensitivity of the sterol-like molecules compared to other extraction methods [47].

To our knowledge, this study is the first report of the parallel quantification of nine steroids directly from killifish tissues of different ages. The comparison between young and old killifish indicates a dysregulation of steroid levels across multiple tissues with age $[10,48,49]$. We observed a reduction in the levels of intermediates of the cholesterol biosynthesis
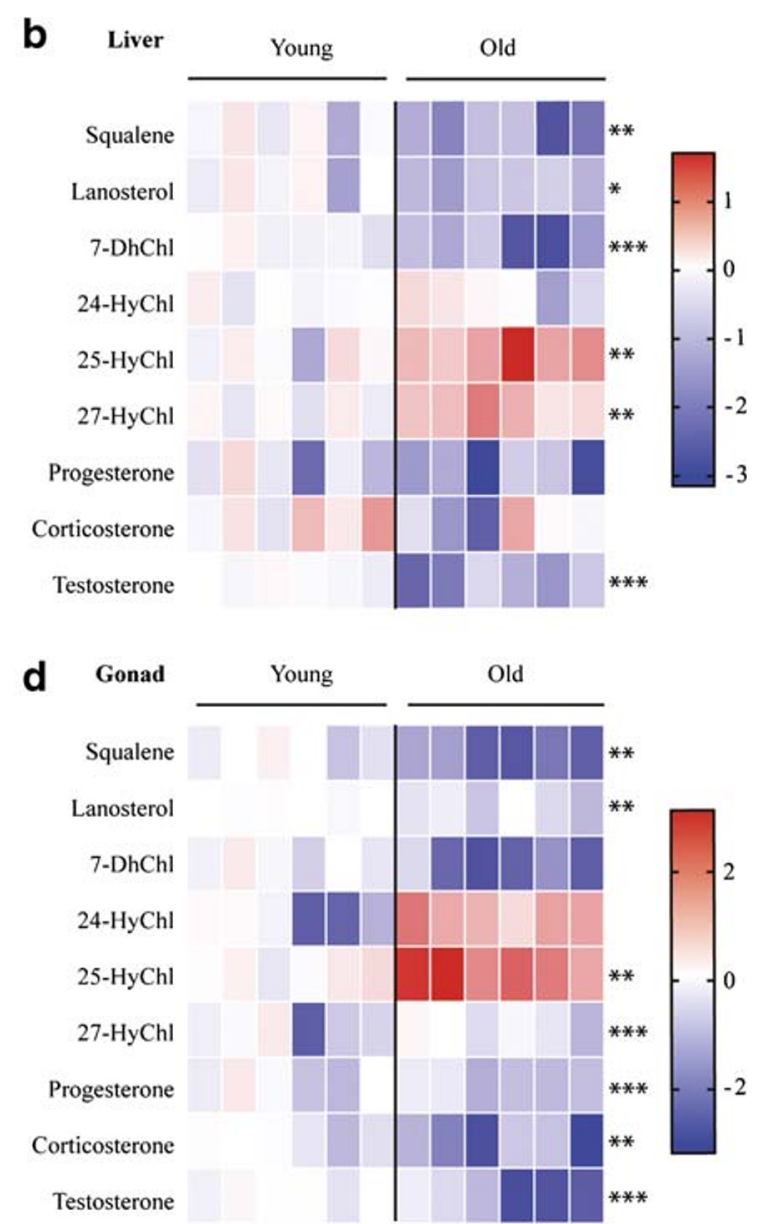

are coloured red and low concentrations are coloured blue. Each column represents a single individual. Original data are reported as histograms in the ESM Figs. S1-S4. Statistical tests were performed using $t$ test. $* P \leq$ $0.05, * * P \leq 0.01, * * * P \leq 0.001$

pathway such as squalene, lanosterol, and 7 Deyhdrocholesterol. These findings are in line with reports that the activity of the sterol regulatory element-binding protein (SREBP) transcription factors, which activate the transcription of enzymes involved in the cholesterol biosynthesis pathway, decrease due to reduced mTORC1 activity during ageing [50-52]. Unfortunately, due to the extraction method and ionization techniques used we were not able to measure free cholesterol concurrently with other steroids as it requires different ionization and instrument set up [53].

Oxysterols are proposed biomarkers of several pathophysiological conditions, such as obesity, atherosclerosis and are involved in immune and inflammatory processes, bone marrow homeostasis and nervous system development [54-56]. Consistently, their levels change during ageing as they impinge on various essential cellular pathways. In line with previous experiments, we show that oxysterol levels are altered during ageing. It has been reported that 24 Hydroxycholesterol levels are decreased in Huntington's disease (HD) and late AD patients [57]. The reduction in 24 
Hydroxycholesterol levels in the brain of aged fish, which we show here, is expected since 24 Hydroxycholesterol levels in the brain are a marker for the metabolic activity of neurons and also the number of neurons, both of which are known to decrease with age [9].

In contrast, it has been shown that levels of 25 and 27 Hydroxycholesterol increase during ageing, particularly in the eye lenses of aged humans, causing cataracts. Additionally, 27 Hydroxycholesterol levels are elevated in atherosclerosis patients and breast tumour cells [58]. The 27 Hydroxycholesterol accumulation in gonad and liver could result from an age-related reduction in CYP27A1 levels, which is the enzyme responsible for its biosynthesis [59].

Ageing is well known to be accompanied by decreasing levels of sex steroid hormones. Our results further support this finding, as both progesterone and testosterone decrease with age in killifish. In men and in rats, a decrease of testosterone levels during ageing is associated with decreasing supply of steroid precursors by Leydig cells [60]. This age-related decline in testosterone steroidogenesis is consistent with decreased mitochondrial expression of steroidogenic acute regulatory protein (StAR) and steroidogenic CYP450s (e.g.CYP11A1) in old versus young rats [61]. We additionally identify estradiol in few tissues, though they were far below the limit of quantification as we used a cohort of only male fish [62].

Taken together, these findings suggest that sex hormones and oxysterols can be developed as biomarkers of ageing and perhaps age-related disease. Further studies will shed light on steroidogenesis and sex hormone production in the newly established model killifish and will reveal new potential endocrine regulators of ageing and ageing-related processes.

\section{Conclusion}

In the current study we thus established a highly sensitive mass spectrometry-based method as a novel tool to study the role of steroids during the ageing process. Using the onevariable-at-time strategy is a viable approach to investigate potential interactions between mass spectrometry parameters. These findings emphasize the importance of thorough parameter optimization during method development.

In general, it is of great importance to find biomarkers of ageing to monitor pathology and age-related diseases. In this regard, it will be interesting in the future to evaluate the possible correlation of steroid concentrations in killifish and ageing. We are confident that this method can be potentially used to investigate sterols in different model organisms but ultimately to improve the steroid detection and quantification in human tissues.
Furthermore, it would be important to assess how genetically or pharmacologically altered steroid quantities alter animal longevity in order to truly understand their role in ageing.

Acknowledgements We thank Dr. Orsolya Symmons for proofreading the manuscript.

Funding information Open access funding provided by Projekt DEAL. This work was financially supported by the Max Planck Society (MaxPlanck-Geselleschaft).

\section{Compliance with ethical standards}

The fish husbandry was performed in the fish facility of the MPI Age (Permit Nr. 576.1.36.6.G12/18 Be); the use of the animals in these trials complied with national legislation in accordance with the ethical standards of the Animal Welfare Body of the MPI Age.

Conflict of interest The authors declare that they have no conflicts of interest.

Open Access This article is licensed under a Creative Commons Attribution 4.0 International License, which permits use, sharing, adaptation, distribution and reproduction in any medium or format, as long as you give appropriate credit to the original author(s) and the source, provide a link to the Creative Commons licence, and indicate if changes were made. The images or other third party material in this article are included in the article's Creative Commons licence, unless indicated otherwise in a credit line to the material. If material is not included in the article's Creative Commons licence and your intended use is not permitted by statutory regulation or exceeds the permitted use, you will need to obtain permission directly from the copyright holder. To view a copy of this licence, visit http://creativecommons.org/licenses/by/4.0/.

\section{References}

1. Berkowitz ML. Detailed molecular dynamics simulations of model biological membranes containing cholesterol. Biochim Biophys Acta. 2009;1788(1):86-96.

2. Schiffer L, Barnard L, Baranowski ES, Gilligan LC, Taylor AE, Arlt W, et al. Human steroid biosynthesis, metabolism and excretion are differentially reflected by serum and urine steroid metabolomes: a comprehensive review. J Steroid Biochem Mol Biol. 2019;105439.

3. Alphonse PAS, Jones PJH. Revisiting human cholesterol synthesis and absorption: the reciprocity paradigm and its key regulators. Lipids. 2016;51(5):519-36.

4. McDonald JG, Smith DD, Stiles AR, Russell DW. A comprehensive method for extraction and quantitative analysis of sterols and secosteroids from human plasma. J Lipid Res. 2012;53(7):1399409.

5. Brown AJ, Jessup W. Oxysterols: sources, cellular storage and metabolism, and new insights into their roles in cholesterol homeostasis. Mol Asp Med. 2009;30(3):111-22.

6. Zmyslowski A, Szterk A. Oxysterols as a biomarker in diseases. Clin Chim Acta. 2019;491:103-13.

7. Ruthirakuhan M, Herrmann N, Andreazza AC, Verhoeff N, Gallagher D, Black SE, et al. 24S-Hydroxycholesterol is associated with agitation severity in patients with moderate-to-severe 
Alzheimer's disease: analyses from a clinical trial with nabilone. J Alzheimers Dis. 2019.

8. Revilla G, Pons MP, Baila-Rueda L, Garcia-Leon A, Santos D, Cenarro A, et al. Cholesterol and 27-hydroxycholesterol promote thyroid carcinoma aggressiveness. Sci Rep. 2019;9(1):10260.

9. Testa G, Staurenghi E, Zerbinati C, Gargiulo S, Iuliano L, Giaccone $\mathrm{G}$, et al. Changes in brain oxysterols at different stages of Alzheimer's disease: their involvement in neuroinflammation. Redox Biol. 2016;10:24-33.

10. Maliković J, Feyissa DD, Kalaba P, Marouf BS, Höger H, Hartmann MF, et al. Age and cognitive status dependent differences in blood steroid and thyroid hormone concentrations in intact male rats. Behav Brain Funct. 2019;15(1):10.

11. Walther A, Philipp M, Lozza N, Ehlert U. The rate of change in declining steroid hormones: a new parameter of healthy aging in men? Oncotarget. 2016;7(38):60844-57.

12. Russell SJ, Kahn CR. Endocrine regulation of ageing. Nat Rev Mol Cell Biol. 2007;8(9):681-91.

13. Guillemot-Legris O, Mutemberezi V, Muccioli GG. Oxysterols in metabolic syndrome: from bystander molecules to bioactive lipids. Trends Mol Med. 2016;22(7):594-614.

14. Sottero B, Rossin D, Staurenghi E, Gamba P, Poli G, Testa G. Omics analysis of oxysterols to better understand their pathophysiological role. Free Radic Biol Med. 2019.

15. Helmschrodt C, Becker S, Schroter J, Hecht M, Aust G, Thiery J, et al. Fast LC-MS/MS analysis of free oxysterols derived from reactive oxygen species in human plasma and carotid plaque. Clin Chim Acta. 2013;425:3-8.

16. Griffiths WJ, Wang Y. Analysis of oxysterol metabolomes. Biochim Biophys Acta. 2011;1811(11):784-99.

17. Cellerino A, Valenzano DR, Reichard M. From the bush to the bench: the annual Nothobranchius fishes as a new model system in biology. Biol Rev Camb Philos Soc. 2016;91(2):511-33.

18. Cellerino A, Terzibasi Tozzini E. Biology of aging: new models, new methods. Semin Cell Dev Biol. 2017;70:98.

19. Platzer M, Englert C. Nothobranchius furzeri: a model for aging research and more. Trends Genet. 2016;32(9):543-52.

20. Ripa R, Dolfi L, Terrigno M, Pandolfini L, Savino A, Arcucci V, et al. MicroRNA miR-29 controls a compensatory response to limit neuronal iron accumulation during adult life and aging. BMC Biol. 2017;15(1):9.

21. Cellerino A, Ori A. What have we learned on aging from omics studies? Semin Cell Dev Biol. 2017;70:177-89.

22. Diotel N, Do Rego JL, Anglade I, Vaillant C, Pellegrini E, Gueguen $\mathrm{MM}$, et al. Activity and expression of steroidogenic enzymes in the brain of adult zebrafish. Eur J Neurosci. 2011;34(1):45-56.

23. Petersen LH, Hala D, Carty D, Cantu M, Martinovic D, Huggett DB. Effects of progesterone and norethindrone on female fathead minnow (Pimephales promelas) steroidogenesis. Environ Toxicol Chem. 2015;34(2):379-90.

24. Lohr H, Hammerschmidt M. Zebrafish in endocrine systems: recent advances and implications for human disease. Annu Rev Physiol. 2011;73:183-211.

25. Dantas NM, Sampaio GR, Ferreira FS, Labre Tda S, Torres EA, Saldanha T. Cholesterol oxidation in fish and fish products. J Food Sci. 2015;80(12):R2627-39.

26. Nyakubaya VT, Durney BC, Ellington MC, Kantes AD, Reed PA, Walter SE, et al. Quantification of circulating steroids in individual zebrafish using stacking to achieve nanomolar detection limits with capillary electrophoresis and UV-visible absorbance detection. Anal Bioanal Chem. 2015;407(23):6985-93.

27. Felix AS, Faustino AI, Cabral EM, Oliveira RF. Noninvasive measurement of steroid hormones in zebrafish holding-water. Zebrafish. 2013;10(1):110-5.

28. Magnisali P, Dracopoulou M, Mataragas M, Dacou-Voutetakis A, Moutsatsou P. Routine method for the simultaneous quantification of 17alpha-hydroxyprogesterone, testosterone, dehydroepiandrosterone, androstenedione, cortisol, and pregnenolone in human serum of neonates using gas chromatography-mass spectrometry. J Chromatogr A. 2008;1206(2):166-77.

29. Budzinski H, Devier MH, Labadie P, Togola A. Analysis of hormonal steroids in fish plasma and bile by coupling solid-phase extraction to GC/MS. Anal Bioanal Chem. 2006;386(5):1429-39.

30. Wear JE, Owen LJ, Duxbury K, Keevil BG. A simplified method for the measurement of urinary free cortisol using LC-MS/MS. J Chromatogr B Anal Technol Biomed Life Sci. 2007;858(1-2):2731.

31. Grebe SK, Singh RJ. LC-MS/MS in the clinical laboratory - where to from Here? Clin Biochem Rev. 2011;32(1):5-31.

32. Ke Y, Gonthier R, Isabelle M, Bertin J, Simard JN, Dury AY, et al. A rapid and sensitive UPLC-MS/MS method for the simultaneous quantification of serum androsterone glucuronide, etiocholanolone glucuronide, and androstan-3alpha, 17beta diol 17-glucuronide in postmenopausal women. J Steroid Biochem Mol Biol. 2015;149: $146-52$.

33. Jeanneret F, Tonoli D, Rossier MF, Saugy M, Boccard J, Rudaz S. Evaluation of steroidomics by liquid chromatography hyphenated to mass spectrometry as a powerful analytical strategy for measuring human steroid perturbations. J Chromatogr A. 2016;1430:97112.

34. Annibal A, Karalay Ö, Latza C, Antebi A. A novel EI-GC/MS method for the accurate quantification of anti-aging compound oleoylethanolamine in C. elegans. Anal Methods. 2018;10(22): 2551-9.

35. Annibal A, Tam H, Latza C, Antebi A. Comparison of ESI-MS/MS and APCI-MS methods for the quantification of folic acid analogs in C. elegans. J Mass Spectrom. 2019;54(4):316-27.

36. Abdel-Khalik J, Bjorklund E, Hansen M. Development of a solid phase extraction method for the simultaneous determination of steroid hormones in H295R cell line using liquid chromatographytandem mass spectrometry. J Chromatogr B Anal Technol Biomed Life Sci. 2013;935:61-9.

37. Raff H, Auchus RJ, Findling JW, Nieman LK. Urine free cortisol in the diagnosis of Cushing's syndrome: is it worth doing and, if so, how? J Clin Endocrinol Metab. 2015;100(2):395-7.

38. Santen RJ, Demers L, Ohorodnik S, Settlage J, Langecker P, Blanchett D, et al. Superiority of gas chromatography/tandem mass spectrometry assay (GC/MS/MS) for estradiol for monitoring of aromatase inhibitor therapy. Steroids. 2007;72(8):666-71.

39. Couchman L, Vincent RP, Ghataore L, Moniz CF, Taylor NF. Challenges and benefits of endogenous steroid analysis by LCMS/MS. Bioanalysis. 2011;3(22):2549-72.

40. Lubin A, Bajic S, Cabooter D, Augustijns P, Cuyckens F. Atmospheric pressure ionization using a high voltage target compared to electrospray ionization. J Am Soc Mass Spectrom. 2017;28(2):286-93.

41. Leinonen A, Kuuranne T, Kostiainen R. Liquid chromatography/ mass spectrometry in anabolic steroid analysis-optimization and comparison of three ionization techniques: electrospray ionization, atmospheric pressure chemical ionization and atmospheric pressure photoionization. J Mass Spectrom. 2002;37(7):693-8.

42. Kalli A, Smith GT, Sweredoski MJ, Hess S. Evaluation and optimization of mass spectrometric settings during data-dependent acquisition mode: focus on LTQ-Orbitrap mass analyzers. J Proteome Res. 2013;12(7):3071-86.

43. Kharchenko A, Vladimirov G, Heeren RM, Nikolaev EN. Performance of Orbitrap mass analyzer at various space charge and non-ideal field conditions: simulation approach. J Am Soc Mass Spectrom. 2012;23(5):977-87.

44. Szabo PT, Kele Z. Electrospray mass spectrometry of hydrophobic compounds using dimethyl sulfoxide and dimethylformamide as solvents. Rapid Commun Mass Spectrom. 2001;15(24):2415-9. 
45. Michalski A, Damoc E, Hauschild JP, Lange O, Wieghaus A, Makarov A, et al. Mass spectrometry-based proteomics using Q Exactive, a high-performance benchtop quadrupole Orbitrap mass spectrometer. Mol Cell Proteomics. 2011;10(9):M111.011015.

46. French D. Advances in bioanalytical techniques to measure steroid hormones in serum. Bioanalysis. 2016;8(11):1203-19.

47. Azadmard-Damirchi S, Dutta PC. A single step solid-phase extraction method for complete separation of sterol oxidation products in food lipids. J Chromatogr A. 2009;1216(1):36-42.

48. Zarrouk A, Vejux A, Mackrill J, O'Callaghan Y, Hammami M, O'Brien N, et al. Involvement of oxysterols in age-related diseases and ageing processes. Ageing Res Rev. 2014;18:148-62.

49. Schroepfer GJ Jr. Oxysterols: modulators of cholesterol metabolism and other processes. Physiol Rev. 2000;80(1):361-554.

50. Porstmann T, Santos CR, Griffiths B, Cully M, Wu M, Leevers S, et al. SREBP activity is regulated by mTORC1 and contributes to Akt-dependent cell growth. Cell Metab. 2008;8(3):224-36.

51. Peterson TR, Sengupta SS, Harris TE, Carmack AE, Kang SA, Balderas E, et al. mTOR complex 1 regulates lipin 1 localization to control the SREBP pathway. Cell. 2011;146(3):408-20.

52. Brohee L, Demine S, Willems J, Arnould T, Colige AC, Deroanne CF. Lipin-1 regulates cancer cell phenotype and is a potential target to potentiate rapamycin treatment. Oncotarget. 2015;6(13):11264 80 .

53. Stanczyk FZ, Clarke NJ. Advantages and challenges of mass spectrometry assays for steroid hormones. J Steroid Biochem Mol Biol. 2010;121(3-5):491-5.

54. Nevius E, Pinho F, Dhodapkar M, Jin H, Nadrah K, Horowitz MC, et al. Oxysterols and EBI2 promote osteoclast precursor migration to bone surfaces and regulate bone mass homeostasis. J Exp Med. 2015;212(11):1931-46.
55. Soroosh P, Wu J, Xue X, Song J, Sutton SW, Sablad M, et al. Oxysterols are agonist ligands of RORgammat and drive Th17 cell differentiation. Proc Natl Acad Sci U S A. 2014;111(33):12163-8.

56. Theofilopoulos S, Wang Y, Kitambi SS, Sacchetti P, Sousa KM, Bodin $\mathrm{K}$, et al. Brain endogenous liver $\mathrm{X}$ receptor ligands selectively promote midbrain neurogenesis. Nat Chem Biol. 2013;9(2):12633.

57. Kreilaus F, Spiro AS, McLean CA, Garner B, Jenner AM. Evidence for altered cholesterol metabolism in Huntington's disease post mortem brain tissue. Neuropathol Appl Neurobiol. 2016;42(6): 535-46.

58. Brown AJ, Jessup W. Oxysterols and atherosclerosis. Atherosclerosis. 1999;142(1):1-28.

59. Glass D, Vinuela A, Davies MN, Ramasamy A, Parts L, Knowles $\mathrm{D}$, et al. Gene expression changes with age in skin, adipose tissue, blood and brain. Genome Biol. 2013;14(7):R75.

60. Ferrini RL, Barrett-Connor E. Sex hormones and age: a crosssectional study of testosterone and estradiol and their bioavailable fractions in community-dwelling men. Am J Epidemiol. 1998;147(8):750-4.

61. Luo L, Chen H, Zirkin BR. Temporal relationships among testosterone production, steroidogenic acute regulatory protein (StAR), and P450 side-chain cleavage enzyme (P450scc) during Leydig cell aging. J Androl. 2005;26(1):25-31.

62. Yamamoto M, Hibi H, Katsuno S, Miyake K. Serum estradiol levels in normal men and men with idiopathic infertility. Int $\mathrm{J}$ Urol. 1995;2(1):44-6.

Publisher's note Springer Nature remains neutral with regard to jurisdictional claims in published maps and institutional affiliations. 